\title{
Transient thermo-solutal convection in a tilted porous enclosure heated from below and salted from above
}

\author{
Fernando J. Guerrero ${ }^{\mathrm{a}, *}$, Rosa Maria Prol-Ledesma ${ }^{\mathrm{a}}$, Nader Karimi ${ }^{\mathrm{b}}$ \\ ${ }^{a}$ Institute of Geophysics, Universidad Nacional Autónoma de México, Cd. Universitaria, \\ México D.F., 04510, México \\ ${ }^{b}$ School of Engineering and Materials Science, Queen Mary University of London, London \\ E1 4 NS, United Kingdom
}

\begin{abstract}
The confinement of $\mathrm{CO}_{2}$ in deep geothermal reservoirs as a means of mitigation of greenhouse gas emissions is continuously motivating research on the retention capacity of these deep aquifers. An important physical containment mechanism is related with $\mathrm{CO}_{2}$ dissolution and thermo-solutal convection. In this context, numerical simulations are performed in this work to assess the effect of inclination, Rayleigh number, and buoyancy ratio on the convective transport in a rectangular porous medium. The porous enclosure is heated from below and cooled from above, whereas a solute is dissolved through the upper boundary with a constant concentration condition and no mass loss through the other boundaries. A set of governing parameters is considered in this assessment: two buoyancy ratios with dominant solute buoyant forces (10 and 100), three Rayleigh numbers $(10,50$, and 80$)$, and three inclination angles plus the horizontal case for reference $\left(5^{\circ}, 10^{\circ}\right.$, and $\left.15^{\circ}\right)$. The solution to the problem is based on a Finite Volume method along with fixed point iteration for the coupled differential equations, and a Conjugate Gradient algorithm for the algebraic system. The model is validated and tested under mesh analysis. The numerical results show that the inclination angle has a minor effect on the convective mixing properties of the porous medium in comparison with the Rayleigh number
\end{abstract}

\footnotetext{
${ }^{*}$ Corresponding author. Tel.:+52 5563371826

Email address: fguerrero@igeofisica.unam.mx (Fernando J. Guerrero)
}

Preprint submitted to International Communications in Heat and Mass TransferSeptember 8, 2020 
and the buoyancy ratio. Increasing the angle slightly decreases the mixing rate as a consequence of the formation of preferential flow paths associated with the inclination, these preferential flow paths make mixing less efficient and give rise to zonation of solute concentration.

Keywords: double-diffussive convection, porous medium, Boussinesq approximation, $\mathrm{CO}_{2}$ dissolution.

1 Nomenclature

\section{Greek symbols}

${ }_{3} \quad \alpha \quad$ Thermal diffusivity

${ }_{4} \quad \beta \quad$ Thermal expansion coefficient

${ }_{5} \epsilon \quad$ Normalized porosity $(\phi / \sigma)$

${ }_{6} \quad \Gamma \quad$ Coefficient $-1 / R a$

$7 \quad \mu \quad$ Viscosity

$8 \quad \nu \quad$ Kinematic viscosity

$9 \quad \phi \quad$ Porosity

${ }_{10} \quad \psi \quad$ Stream function

${ }_{11} \quad \rho \quad$ Density

${ }_{12} \sigma \quad$ Ratio of solid-fluid heat capacities

${ }_{13} \theta \quad$ Inclination angle

14 Other symbols

15 - Overbar denotes dimensional variables and operators

16 Roman letters

${ }_{17} \quad \mathbf{e} \quad$ Vector $(\sin \theta, \cos \theta)$ 


\begin{tabular}{|c|c|c|}
\hline 18 & $\mathbf{u}$ & Darcy's velocity \\
\hline 19 & $A$ & Area \\
\hline 20 & $B$ & Height of the porous enclosure \\
\hline 21 & $C$ & Width of the porous enclosure \\
\hline 22 & $D$ & Mass diffusivity \\
\hline 23 & $g$ & Gravitational constant \\
\hline 24 & $k$ & Permeability \\
\hline & Le & Lewis number \\
\hline 26 & $N$ & Buoyancy ratio \\
\hline & $N u$ & Nusselt number \\
\hline & $P$ & Pressure \\
\hline 20 & $R a$ & Rayleigh number \\
\hline 30 & $S$ & Solute concentration \\
\hline & $S_{a}$ & Average concentration \\
\hline 32 & $S h$ & Sherwood number \\
\hline 33 & $T$ & Temperature \\
\hline 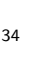 & $t$ & Time \\
\hline 35 & $x, y$ & Cartesian coordinates \\
\hline & Subsc & ripts \\
\hline 37 & $0, c, r$ & Reference values \\
\hline 38 & $a$ & Average value \\
\hline 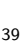 & $S$ & Solutal \\
\hline & $T$ & Thermal \\
\hline
\end{tabular}




\section{Introduction}

Double-diffusive convection in porous media is a major process for the physical containment of $\mathrm{CO}_{2}$ in deep aquifers $[1,2]$. The problem arises from the fact that supercritical $\mathrm{CO}_{2}$ injected in a confined aquifer accumulates beneath the cap rock, where dissolution of $\mathrm{CO}_{2}$ in water takes place over time [3], this leads to convective mixing due to a slightly higher density of the $\mathrm{CO}_{2}$-Brine solution. Moreover, the $\mathrm{CO}_{2}$-Brine interface might not be horizontal, but present some degree of inclination due to structural conditions and $\mathrm{CO}_{2}$-front displacement. We address the dissolution process of a solute in this situation.

It is worth mentioning that this topic is relevant in other scientific and engineering fields, such as: evolution of hydrothermal systems [4], materials manufacturing [5], and nuclear waste repository [6].

Fundamental aspects of double-diffusive convection have been stated by several authors in the past. Early work was presented by Nield [7], who addressed the onset of convection in a porous layer heated and salted from below. On the fact that thermal diffusions occurs more rapidly than solute diffusion, he pointed out that the onset of convention can be characterized either by monotonic or oscillatory instability depending on whether the solute gradients enhance or counteract the instability associated with thermal gradients. Taunton et al. [8] extended the stability analysis of this problem and pointed out that concentration density differences are more effective in promoting instabilities. Trevisan and Bejan [9] addressed the problem of steady-state solutions at high Rayleigh numbers (up to 2000), their work revealed that the Sherwood number relates to the governing parameters $R a$ and $L e$ with three distinct scaling laws. Rosenberg and Spera [10] conducted numerical simulations in order to find relations between $N u$ and $S h$ with the governing parameters $R a, L e$, and $N$ in steady-state solutions. They also evaluated the effect of buoyancy ratio $N$ on the dynamics of transient convection at $R a=600$. The results showed that $N u$ follows an oscillatory behavior as the flow develops and becomes more complex for greater $N$. 
Subsequent work was concerned with a variety of boundary conditions and configurations. Lin [11] addressed this problem considering the lateral walls of the porous enclosure as the source of thermal and concentration gradients, with adiabatic and impervious top and bottom boundaries. In a similar configuration, Mamou et al. [12] looked into the existence of multiple steady-state solutions (a condition which is known from purely thermal free convection $[13,14]$ ), they obtained governing parameters that allow different convective modes. Assuming the same kind of boundary conditions, Mamou et al. [15] presented the stability analysis for the particular case of opposing buoyancy forces $(N=-1)$, they reported parametric relations $(L e, \bar{\epsilon}, A)$ with the onset of stationary and oscillatory convection. A three-dimensional version of this problem was presented by Sezai and Mohamad [16] with the additional assumption of a Darcy-Brinkman flow model. They identified steady-state solutions for a set of governing parameters $(R a, L e$, and $N)$ and found that in the case of opposing buoyancy forces the convective mode is strictly 3D. Nithiarasu et al. [17], considered the case of prescribed temperature and concentration in a lateral boundary, and convective heat and mass transfer in the opposite, giving rise to the Biot number as a governing parameter of the system. Zhao et al. [18] considered a porous medium heated and salted from a segment of a lateral boundary keeping the opposite boundary at constant temperature and concentration, and top and bottom boundaries as adiabatic and impermeable. Their work is particularly important to understand the transport behavior associated with localized heat and mass sources. In a later model, the use of the Darcy-Brinkman equation allowed the evaluation of the no-slip boundary condition on a solid wall [19]. Coupled porous medium and free fluid systems, as well as tilted porous enclosures have also been studied in relation with these boundary conditions [20, 21].

With regard to dissolution processes from the top boundary, theoretical studies have determined the critical conditions for the onset convective mixing as well as mixing evolution in absence of thermal gradients $[22,23,24]$. The problem is stated as the stability of a diffusive boundary layer in a semi-infinite domain [22], which leads to the definition of a critical time and wave number 
for the onset of convection, both proportional to the ratio between diffusive and buoyancy forces. Further, the mixing has been described in terms of five stages, comprising diffusion, onset of instability, onset of convection (fingering), merging of convective fingers, and convective shutdown [3]. Concerning systems heated from below and salted from above, Islam et al. [2] described the general aspects of the convective transport, characterized by fingering and merging. They use the average concentration of the solute in the porous medium as a parameter to measure how the thermal and solute Rayleigh numbers enhance solute transport with time. They also evaluated the aspect ratio of the porous enclosure and found that wide is more advantageous for solute transport than tall. Anisotropy effects, geochemical reactions, presence of impermeable layers, and more recently, external forces due to fluid injection in the porous medium have also been examined [25, 26, 27, 28].

Even though a variety of general aspects of this problem are now available in the literature, we consider that the effect of inclination angles has received less attention. In particular, the extent to which the tilt angle accelerates or delays the convection and mixing has not been addressed in the context of this problem. For this reason we present in this paper numerical simulations with a focus on the inclination of the porous medium to provide answers to this particular question.

\section{Problem formulation}

The problem concerns a rectangular porous enclosure of height $B$ and length $C$ with impermeable walls and fully saturated with an incompressible fluid (Fig. 1 ). It is assumed a constant aspect ratio of the enclosure $C / B=3$. The medium is heated from below at constant temperature, and salted from above at a constant concentration. Initially the medium is at constant temperature $\bar{T}_{c}$ and in absence of dissolved solute $(\bar{S}=0)$. The lateral boundaries are adiabatic and the enclosure is inclined an angle $\theta$ with respect to the horizontal. The basic assumptions for this problem include local thermal equilibrium, constant poros- 


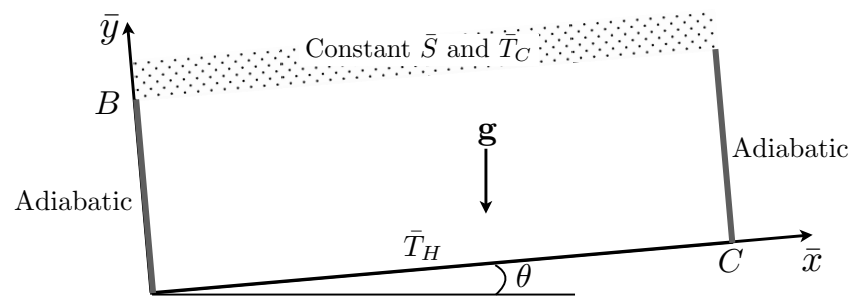

Figure 1: Schematic model of a rectangular porous enclosure tilted an angle $\theta$. The enclosure is heated from its base and cooled form the top at constant temperatures, whereas dissolution occurs at constant concentration on the top boundary.

ity, fluid flow is described by Darcy's law, and the Boussinesq approximation can be applied. From these considerations the momentum equation can read as follows:

$$
\overline{\mathbf{u}}=-\frac{k}{\mu}(\bar{\nabla} \bar{P}+\rho g \mathbf{e})
$$

where the vector $\mathbf{e}=(\sin \theta, \cos \theta)$ determines the components of the buoyancy force as a function of the inclination angle. The heat transfer equation takes the form $[29]$ :

$$
\sigma \frac{\partial \bar{T}}{\partial \bar{t}}+\overline{\mathbf{u}} \cdot \bar{\nabla} \bar{T}=\bar{\nabla} \cdot(\alpha \bar{\nabla} \bar{T}),
$$

likewise, the mass transfer equation reads:

$$
\phi \frac{\partial \bar{S}}{\partial \bar{t}}+\overline{\mathbf{u}} \cdot \bar{\nabla} \bar{S}=\bar{\nabla} \cdot(D \bar{\nabla} \bar{S}) .
$$

The Boussinesq approximation leads to the condition of incompressibility:

$$
\bar{\nabla} \cdot \overline{\mathbf{u}}=0
$$

furthermore, an equation of state for the buoyancy term is required [29]:

$$
\rho=\rho_{0}\left[1-\beta_{T}\left(\bar{T}-\bar{T}_{0}\right)-\beta_{S}\left(\bar{S}-\bar{S}_{0}\right)\right]
$$

The pressure $\bar{P}$ (Eq. 1) is defined so that it is taken relative to the hydrostatic pressure $\rho_{0} g z$, this leads to the common form to the Darcy momentum equation with the buoyancy term: 


$$
\overline{\mathbf{u}}=-\frac{k}{\mu}\left(\bar{\nabla} \bar{P}-\left(\beta_{T}\left(\bar{T}-\bar{T}_{0}\right)+\beta_{S}\left(\bar{S}-\bar{S}_{0}\right)\right) \rho_{0} g \mathbf{e}\right)
$$

The following dimensionless parameters and operators are introduced for the formulation of the mathematical problem:

$$
\begin{gathered}
x=\frac{\bar{x}}{B}, \quad y=\frac{\bar{y}}{B}, \quad t=\frac{\bar{t} \alpha}{\sigma B^{2}}, \quad \mathbf{u}=\frac{B}{\alpha}(\bar{u}, \bar{v}), \quad P=\frac{k}{\mu \alpha} \bar{P}, \\
T=\frac{\bar{T}-\bar{T}_{0}}{\Delta \bar{T}}, \quad S=\frac{\bar{S}-\bar{S}_{0}}{\Delta \bar{S}}, \quad \Delta \bar{T}=\bar{T}_{0}-\bar{T}_{c}, \quad \Delta \bar{S}=\bar{S}_{0}-\bar{S}_{r}, \\
R a=\frac{g B k \beta_{T} \Delta \bar{T}}{\alpha \nu}, \quad L e=\frac{\alpha}{D}, \quad N=\frac{\beta_{S} \Delta \bar{S}}{\beta_{T} \Delta \bar{T}}, \quad \epsilon=\frac{\phi}{\sigma}, \quad \nabla=B \bar{\nabla} .
\end{gathered}
$$

39 Next, we define the Nusselt number, Sherwood number, and the average concentration of the solute as the physical parameters for the analysis of the numerical results:

$$
N u=\int\left|\frac{\partial T}{\partial y}\right|_{y=1} d x, \quad S h=\int\left|\frac{\partial S}{\partial y}\right|_{y=1} d x, \quad S_{a}=\frac{\int S d A}{A} .
$$

The dimensionless equations read:

$$
\begin{gathered}
\frac{\partial T}{\partial t}-\nabla^{2} T+\mathbf{u} \cdot \nabla T=0 \\
\epsilon \frac{\partial S}{\partial t}-\frac{1}{L e} \nabla^{2} S+\mathbf{u} \cdot \nabla S=0 \\
\mathbf{u}+\nabla P=R a(T+N S) \mathbf{e}
\end{gathered}
$$

In what follows, a constant $\epsilon=1$ was assumed in our mathematical model. Next, the momentum equation (Eq. 11) is written in terms of the the stream function, $\psi: u=\partial \psi / \partial y, v=-\partial \psi / \partial y[30,31]$. This leads to a Poisson equation of the form: 


$$
\Gamma \nabla^{2} \psi=\left(\frac{\partial T}{\partial x}+N \frac{\partial S}{\partial x}\right) \cos \theta-\left(\frac{\partial T}{\partial y}+N \frac{\partial S}{\partial y}\right) \sin \theta,
$$

with $\Gamma=-1 / R a$. Equations 9,10 , and 12 represent the mathematical problem to be solved subject to the following boundary conditions:

For the heat transfer equation, the temperature field satisfies

$$
\begin{gathered}
\frac{\partial T}{\partial x}=0, \quad \text { for } \quad x=0 \text { and } \quad x=3 \\
T=1, \quad \text { for } y=0 \text { and } t>0 \\
T=0, \quad \text { for } y=1 \text { and } t>0 .
\end{gathered}
$$

The mass transfer equation is subject to

$$
\begin{aligned}
\frac{\partial S}{\partial x} & =0, \quad \text { for } x=0 \text { and } x=3, \\
\frac{\partial S}{\partial y} & =0, \quad \text { for } y=0, \\
S & =1, \quad \text { for } y=1 \text { and } t>0 .
\end{aligned}
$$

With regard to the momentum equation (Eq. 12), $\psi=0$ is imposed at the boundaries to achieve no fluid flow through the walls:

$$
\begin{aligned}
& \psi=0, \quad \text { for } \quad x=0 \quad \text { and } \quad x=C, \\
& \psi=0, \quad \text { for } \quad y=0 \quad \text { and } \quad y=1 .
\end{aligned}
$$

\section{Methods and solution}

The time-dependent mathematical problem was discretized with the Finite Volume numerical method [32]. The discretization of the convective terms of the heat and mass transfer equations (Eqs. 9 and 10) was done under the upwind scheme, and a first-order fully implicit scheme was used for the temporal term of both equations. A fixed point algorithm was implemented for the solution of the coupled differential equations [33]. Further, the algebraic systems were solved with a Conjugate Gradient algorithm. A convergence criterion of $1 \times 10^{-6}$ was used in both iterative algorithms. The numerical model was implemented 
in Fortran 90 with multithread libraries (OpenMP by Intel ${ }^{\circledR}$ ). The simulations were performed in processors Xeon ${ }^{\circledR}$ E5-2630 v3, $2.40 \mathrm{GHz}$.

A time step $\Delta t=1 \times 10^{-6}$ was selected for the simulations after a calibration process. Likewise, a mesh sensitivity analysis (Sec. 3.1) permitted us to choose a mesh given by $\Delta x=\Delta y=1 / 250$. This mesh turned out a sufficiently high resolution mesh to capture small scale flow features.

\subsection{Validation}

The model was validated by comparison with the results reported by Islam et al. [2]. In this case, the aspect ratio of the porous enclosure is 1 , and $\theta=0$. Additionally, a perturbation of the concentration gradient is introduced as a boundary condition, in order to promote the onset of 'fingering' in the solute transport: $S(x, 1)=1+0.01 \sin (48 \pi x)$. Based on these conditions, three simulations were performed considering fixed $N=-100, L e=1$, a simulation time $t=6.3 \times 10^{-3}$ and three Rayleigh numbers. Figure 2 presents the comparison between our results and the reference. There is consistency in the results despite some differences as the system becomes more convective $(R a=100)$, this slight deviation in the models can be attributed to the different approaches to solve both the system of differential equations and the algebraic systems.

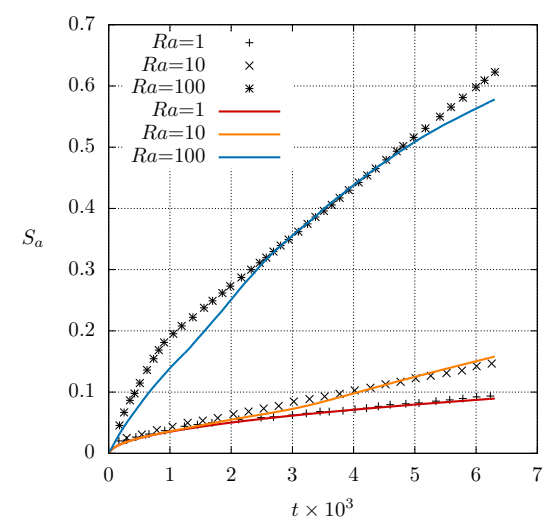

Figure 2: Comparison between the present results (curves) and the reference [2] (points).

The simulation result corresponding to $R a=100$ was further tested under 
two finer meshes: $\Delta x=\Delta y=1 / 500$ and $\Delta x=\Delta y=1 / 1000$, a smaller time step was required, however, to maintain the stability of the solution $(\Delta t=$ $\left.1 \times 10^{-7}\right)$. A comparison of $S_{a}$ after a simulation time $t=6.3 \times 10^{-3}$ show that a small difference is obtained $(\sim 6 \%)$ with the finer meshes with a considerable increase in computing time (Table 1). On this basis $\Delta x=\Delta y=1 / 250$ was preferred as a suitable discretization for the cases studied here, involving a larger aspect ratio and longer simulation times.

Table 1: Mesh sensitivity analysis.

\begin{tabular}{|ccc|}
\hline Mesh & $S_{a}$ & cpu time $(\mathrm{h})$ \\
\hline $1 / 250$ & 0.58 & 0.35 \\
$1 / 500$ & 0.61 & 5.21 \\
$1 / 1000$ & 0.63 & 33.6 \\
\hline
\end{tabular}

\section{Numerical results and discussion}

\subsection{Average concentration}

The average concentration $S_{a}$ is a measure of the degree of saturation of the solute in the fluid. Since the porous medium does not allow mass loss through the boundaries, the average concentration $S_{a}$ tends to 1 in the porous enclosure, which is a saturation condition. This section is intended to quantify the extent to which the governing parameters $N, R a$, and $\theta$ enhance (or delay) mixing whereas $L e$ is set constant at 10. Figure 3 presents the relation of $S_{a}$ for the three parameters examined, each buoyancy ratio is presented in separate graphs (Fig. 3-A and B). The most effective means to enhance mixing turned out to be the magnitude of the buoyancy ratio $(|N|)$. Regardless of $R a$ and $\theta$, for $N=-100, S_{a}$ reaches about $50 \%$ or more at $t=0.05$, while the curves for $N=-1$ present more moderate slopes. At the end of the simulation time ( $t=0.3$ ) the cases $N=-1$ have all of them evolved up to $S_{a}=0.9$ or above.

With regard to $R a$, as expected the curves display a faster mixing for higher $R a$. The buoyant forces of both thermal and solute gradients are increased with 

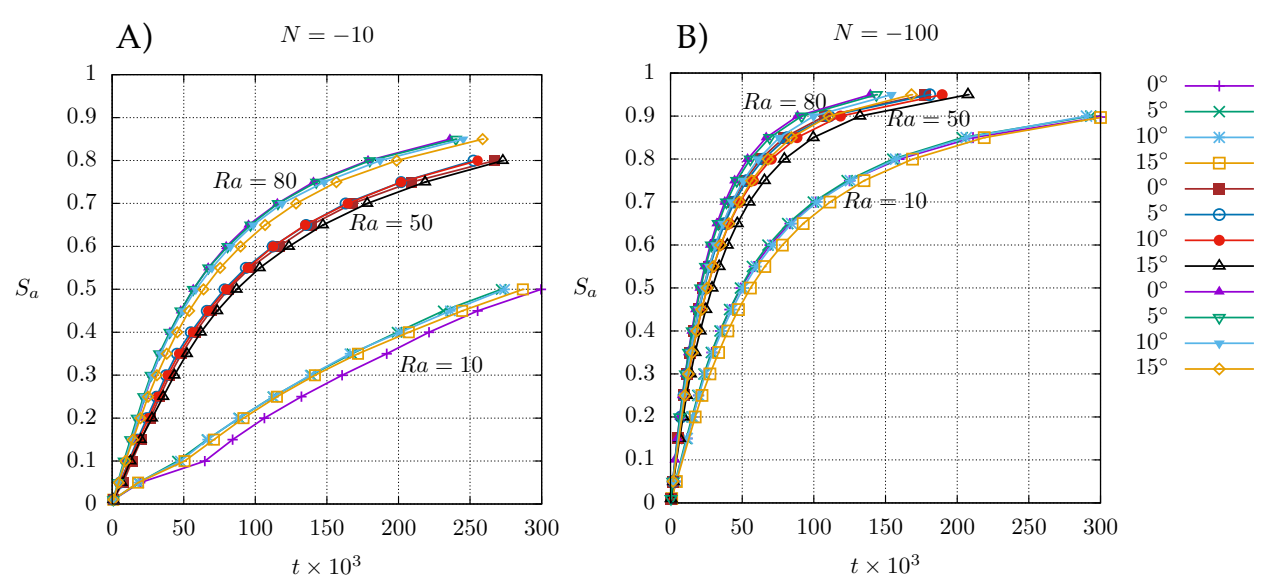

Figure 3: Average concentration $S_{a}$ as a function of the governing parameters $N, R a$, and $\theta$.

$R a$ leading to a highly convective system. An interesting feature is that the curves corresponding to $R a=50$ and $R a=80$ for $N=-100$ form almost a single set of curves and difficult to distinguish between each other. Likewise, the set $R a=10$ appears less distant from $R a=50$ and $R a=80$ than in the case $N=-10$. This behavior is associated with the large magnitude of $N$, that exerts a major control of the convection in the system. This feature is not evident for $N=-10$, where the three sets of curves corresponding with the three Rayleigh numbers are still clearly identified. Consequently, it can be concluded that $R a$ becomes a controlling parameter for moderate and small $N$. This is also expected from the inspection of Equation 12, where the buoyant force for the solute gradient is a multiple of $R a$. Therefore, $R a$ and $N$ are equally important to govern the flow system only when $N=-1$.

Figure 3 further shows that the effect of varying $\theta$ on the evolution of $S_{a}$ is moderate for a given $R a$ and $N$. This effect appears to be negligible in some cases, for instance $N=-100$ and $R a=10$, where the curves $\theta=0^{\circ}, 5^{\circ}$, and $10^{\circ}$ are almost equivalent . Moreover, the largest inclination angle, $15^{\circ}$, does not imply the most convective system. With a single exception $(N=-10$, $R a=10), \theta=15^{\circ}$ delays mixing, even though moderately. Unlike purely ther- 
mal convection, in which the increase of the slope angle (in the same space of parameters) leads to more convection-dominated transport with well defined multicellular convection [14], in this case solutal convection governs the convective transport with a strong dependency on $N$ and more complex convective cell configurations. An inspection of $S_{a}$ at long times shows the weak dependence of this parameter with regard to $\theta$ (Table 2). Even in the most diffusive case $(N=-10, R a=10), S_{a}$ behaves similarly for every angle with differences no greater than $6 \%$.

Table 2: Average concentration $S_{a}$ at the end of the simulation time $t=0.3$.

\begin{tabular}{|c|ccc|ccc|}
\hline & \multicolumn{5}{|c|}{$S_{a}$} \\
\hline & \multicolumn{3}{|c|}{$N=-10$} & \multicolumn{3}{c|}{$N=-100$} \\
\hline$\theta$ & $R a=10$ & $R a=50$ & $R a=80$ & $R a=10$ & $R a=50$ & $R a=80$ \\
\hline $0^{\circ}$ & 0.50 & 0.82 & 0.88 & 0.90 & 0.98 & 0.98 \\
$5^{\circ}$ & 0.53 & 0.83 & 0.88 & 0.90 & 0.98 & 0.98 \\
$10^{\circ}$ & 0.53 & 0.83 & 0.88 & 0.90 & 0.97 & 0.98 \\
$15^{\circ}$ & 0.51 & 0.82 & 0.87 & 0.90 & 0.97 & 0.98 \\
\hline
\end{tabular}

\subsection{Sherwood and Nusselt numbers}

The behavior of the Sherwood number is presented in Figure 4 for limit angles $\left(0^{\circ}\right.$ and $\left.15^{\circ}\right)$. There is a monotonic and steep decrease at the beginning when the transport is diffusive. This decrease lasts until the onset of convective mass transport characterized by fingering, the rebound on $S h$ is then followed by an oscillatory decrease characterized by merging of convective fingers. The decrease will continue until the porous medium is fully saturated. As expected, the time scale of the evolution of $S h$ varies considerably with $N$. For $N=-10$, the development from diffusion to merging of convective fingers takes about $t=0.006$, whereas the same transition occurs for $t<0.001$ in $N=-100$. Concerning the slope angle, it is evident from Figure 4-A and B that the inclination becomes a source of instability for the onset of convective fingers since $\theta=0^{\circ}$ displays a slightly longer diffusive stage. With regard to $N=-100$ the inclination of the porous medium is accompanied by a more complex transition from 

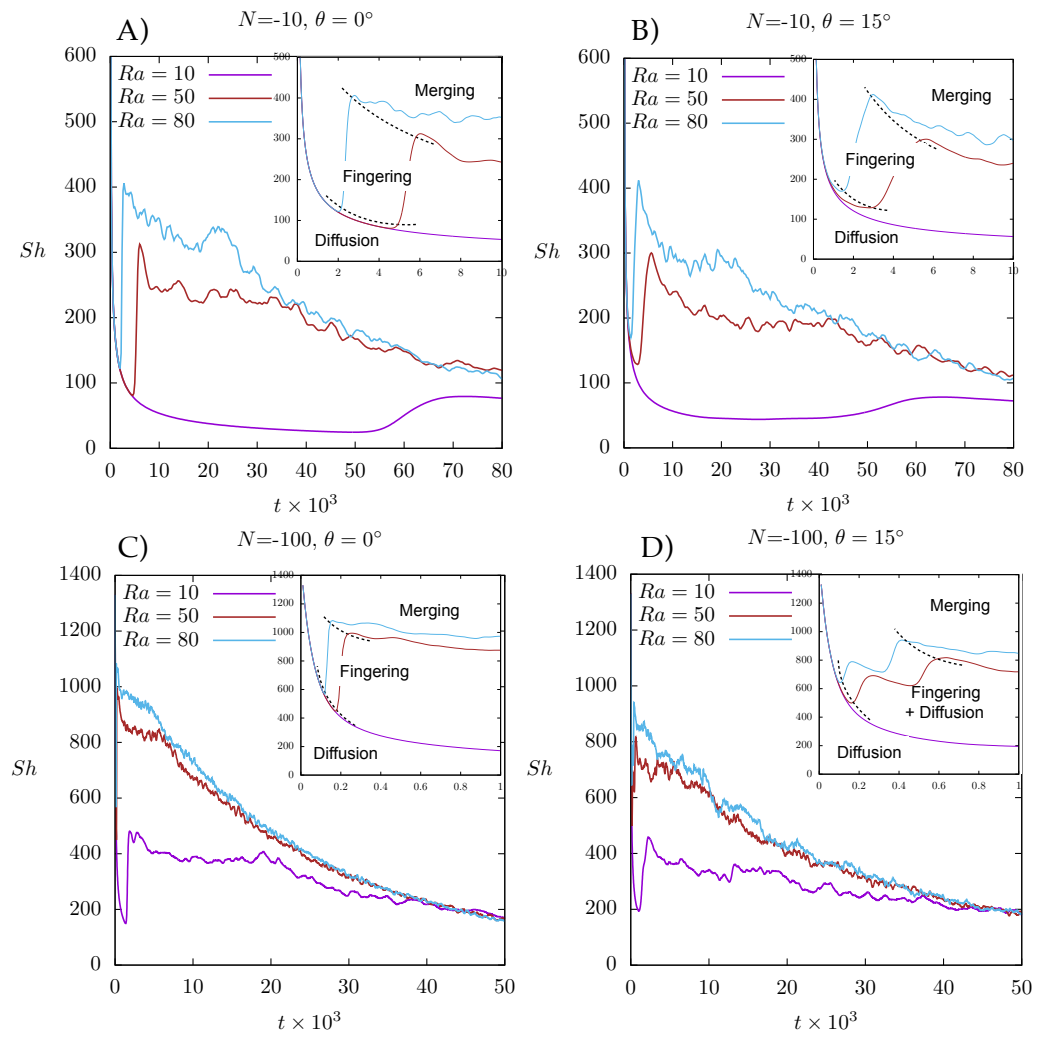

Figure 4: Sherwood number as a function of time for selected angles. The insets show the phases that characterize mass transport.

diffusion to convection, in which part of the upper boundary starts developing convection while the rest of it remains diffusive (Fig. 12), this region is labeled as a Fingering + Diffusion regime in the inset of Figure 4-D.

The evolution of the Nusselt number is in general oscillatory (Figure 5), with the exception of $N=-10, R a=10$, that displays a smoother trend for every $\theta$. The curves present peaks of maximum $N u$ that appear considerably sooner in $N=-100$ (Fig. 5-C and D) in consistency with earlier convective effects associated with high $N$. It is important to highlight that the amplitude of the oscillations of $N u$ decreases over time, which indicates its relation with the mass flux through the boundary. As the fluid approaches saturation the 

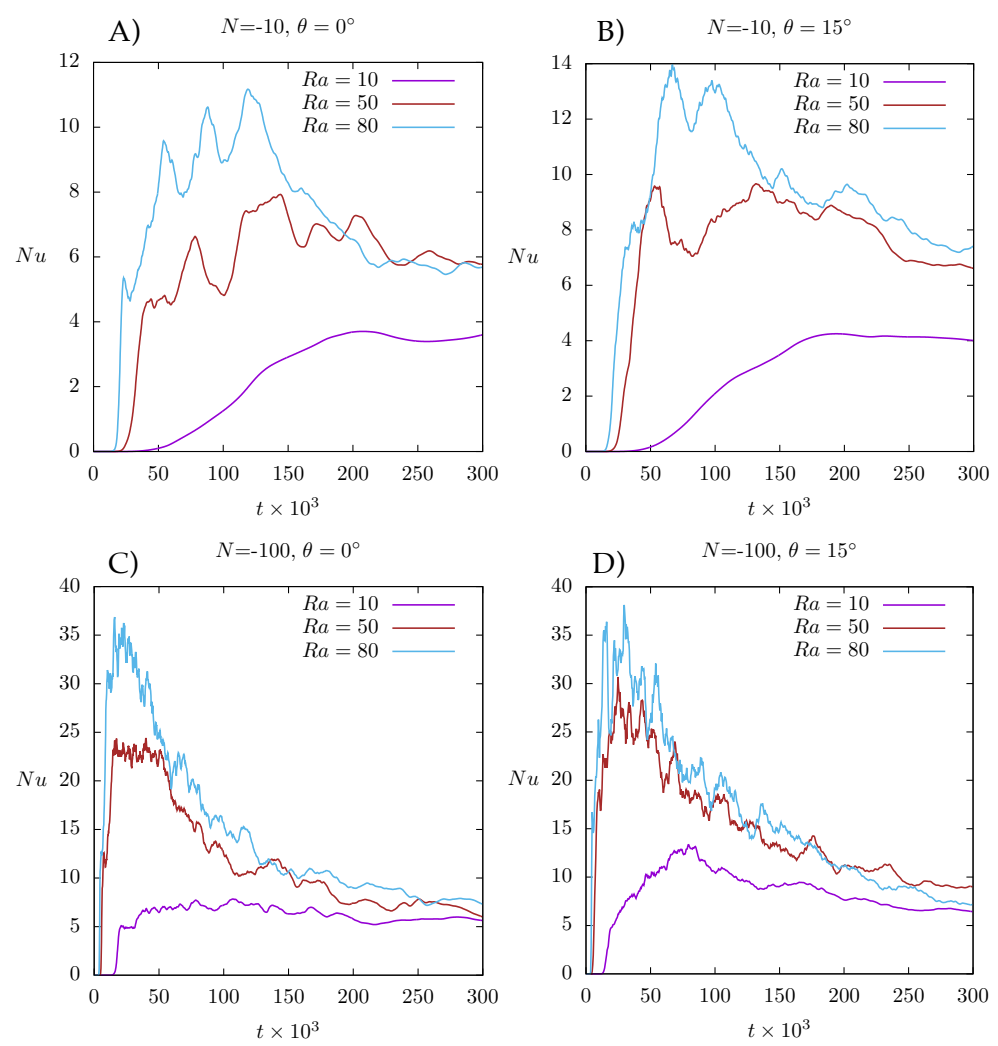

Figure 5: Nusselt number as a function of time for selected angles.

Nusselt number recovers a smoother trend (typical in pure thermal convection) because the mass flux starts vanishing and so do the fingering and merging that strongly and dynamically control transport at the upper boundary. A further evidence that the oscillations of $N u$ in the porous cavity are associated with the convective mass transport resides on the fact that $N u$ is smooth when $S h$ is so (4-A and B).

An alternative way to examine the behavior of $S h$ and $N u$ is integrating them over the simulation time in order to have a measure of the mass and heat fluxes over the entire period (from $t=0$ to $t=0.3$ ). These integrated $S h$ and $N u$ are presented in Figure 6. Two additional Rayleigh numbers $(R a=50$ and $R a=100)$ and a buoyancy ratio $(N=-50)$ are included in order to have wider view of 

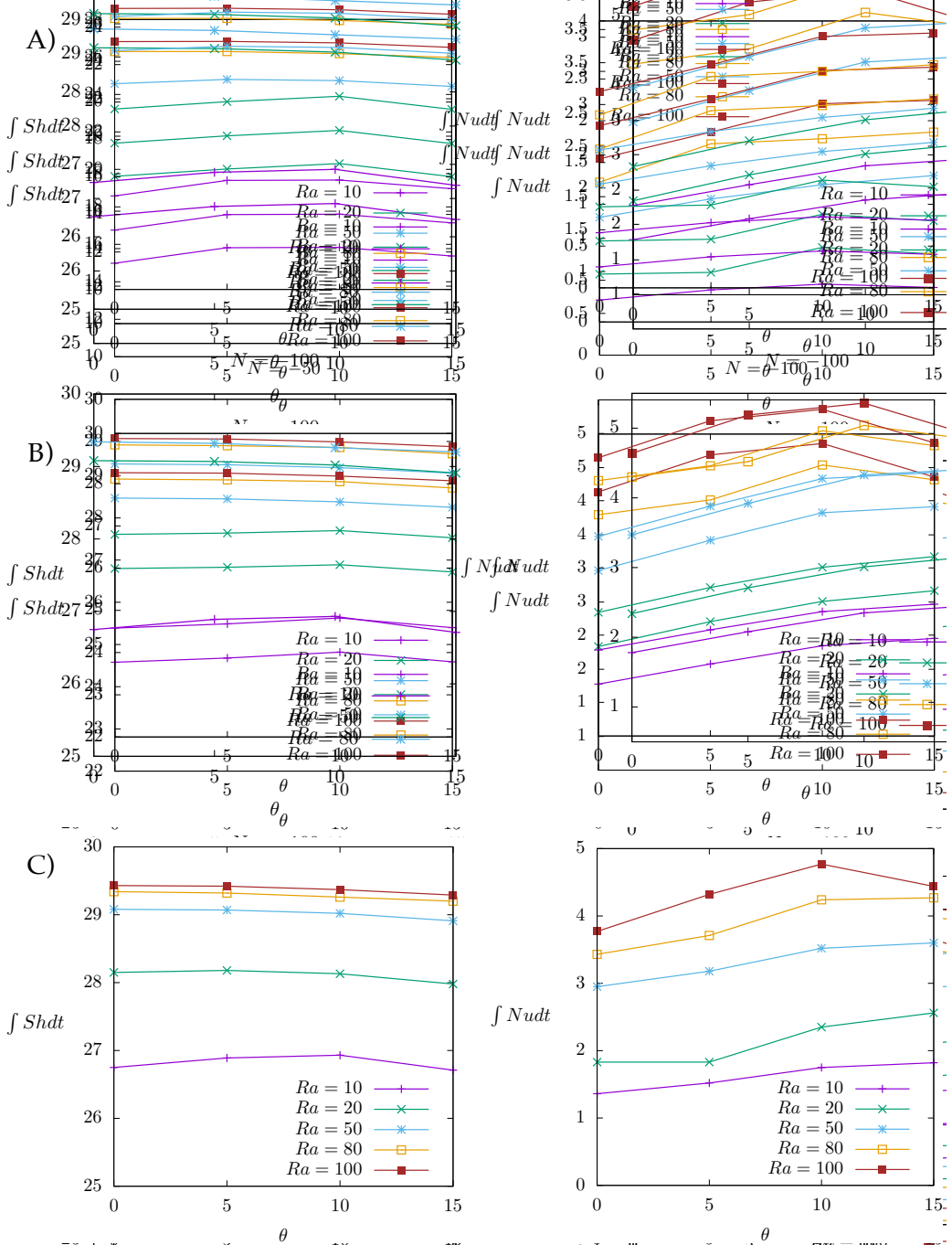

Figure 6: Integrated Sherwood and Nusselt numbers over the entire simulation time as a function of $\theta$ and for $L e=10$ : A) $N=-10$, B) $N=-50$, C) $N=-100$.

this parameter analysis. With respect to $\int S h d t$, there is a small sensitivity to $\theta$, regardless of the buoyancy ratio (as expected from Figure 3). This is particularly evident for high $R a$ which curves display a small decrease with $\theta$, associated with the formation of a preferential flow direction that decreases mass diffusion (Sec. 4.3). Additionally, as $R a$ increases the integrated Sherwood 
number tends to constant value (see for example the small difference between $R a=80$ and $R a=100)$. The vigorous convection associated with high $R a$ leads the system to saturation condition, so that the mass flux turns out almost equivalent.

As regards the Nusselt number (Fig. 6), there is a general trend of favoring the heat transfer increasing $\theta$, in agreement with the case of purely thermal convection. This can be observed in Figure 7 that illustrates the effect of increasing the angle for a given set of governing parameters and the same $t$. For $\theta=15^{\circ}$ the flow pattern tends to adopt a three-cell convective mode with two large upwelling thermal plumes, whereas $\theta=0^{\circ}$ displays a lower magnitude multicellular convection highly conditioned by the convective mass transport.

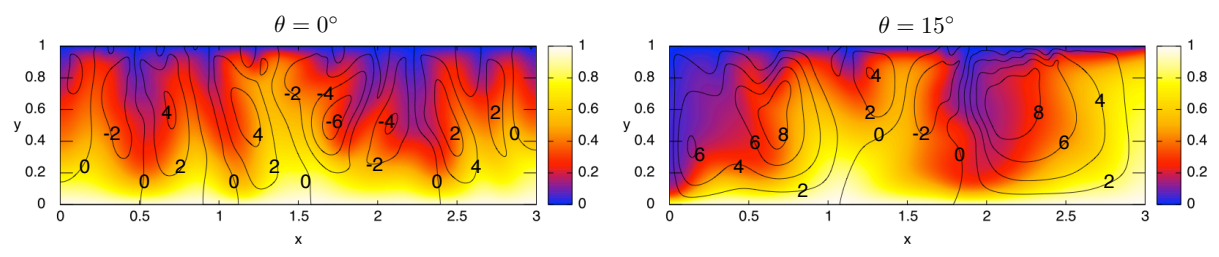

Figure 7: Temperature fields and streamlines for $N=10, R a=50$ at $t=0.15$.

It is convenient to compare this behavior with a different Lewis number. A more moderate case in which mass and thermal diffusivities are of the same order of magnitude $(L e=2)$ is presented in Figure 8. The mass transport $\left(\int S h d t\right)$ presents a consistent behavior with Figure 6, however, smaller values of $\int S h d t$ indicate smaller concentration gradients at the top boundary as a compensation for a higher mass diffusivity (1/Le, Eq. 10). An important exception is the most diffusive case $(R a=10, N=10)$ that displays a monotonic increase with $\theta$, so that as the systems becomes more diffusive the inclination at these moderate angles can enhance mass transport. The integrated Nusselt number, on the other hand, strongly depends on the history of the convective regime, number and intensity of convective cells. Unlike the case $L e=10$ (Fig. 6), it is observed less dependence of $\int N u d t$ on $\theta$ being essentially dependent on $R a$ and $N$. 

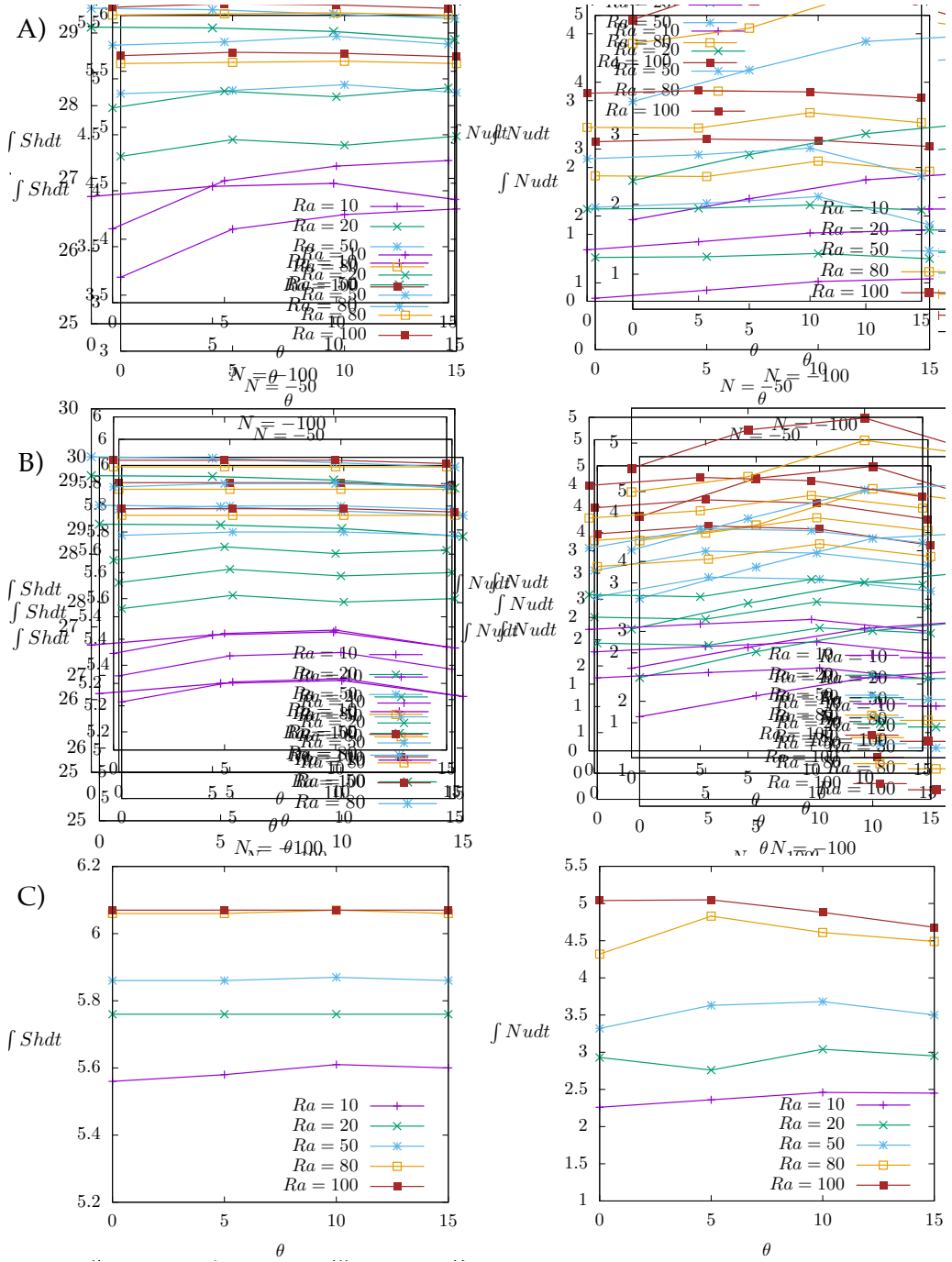

Figure 8: Integrated Sherwood and Nusselt numbers over the entire simulation time as a function of $\theta$ and for $L e=2$ : A) $N=-10$, B) $N=-50$, C) $N=-100$.

\subsection{Transient mass and heat transport}

The most representative convective features are described in this section. Firstly, the case with the lowest buoyancy forces is shown in Figure 9. Diffusive transport progresses faster for the temperature and subsequently the onset of convection takes place on the upper boundary. At the end of the simulation 

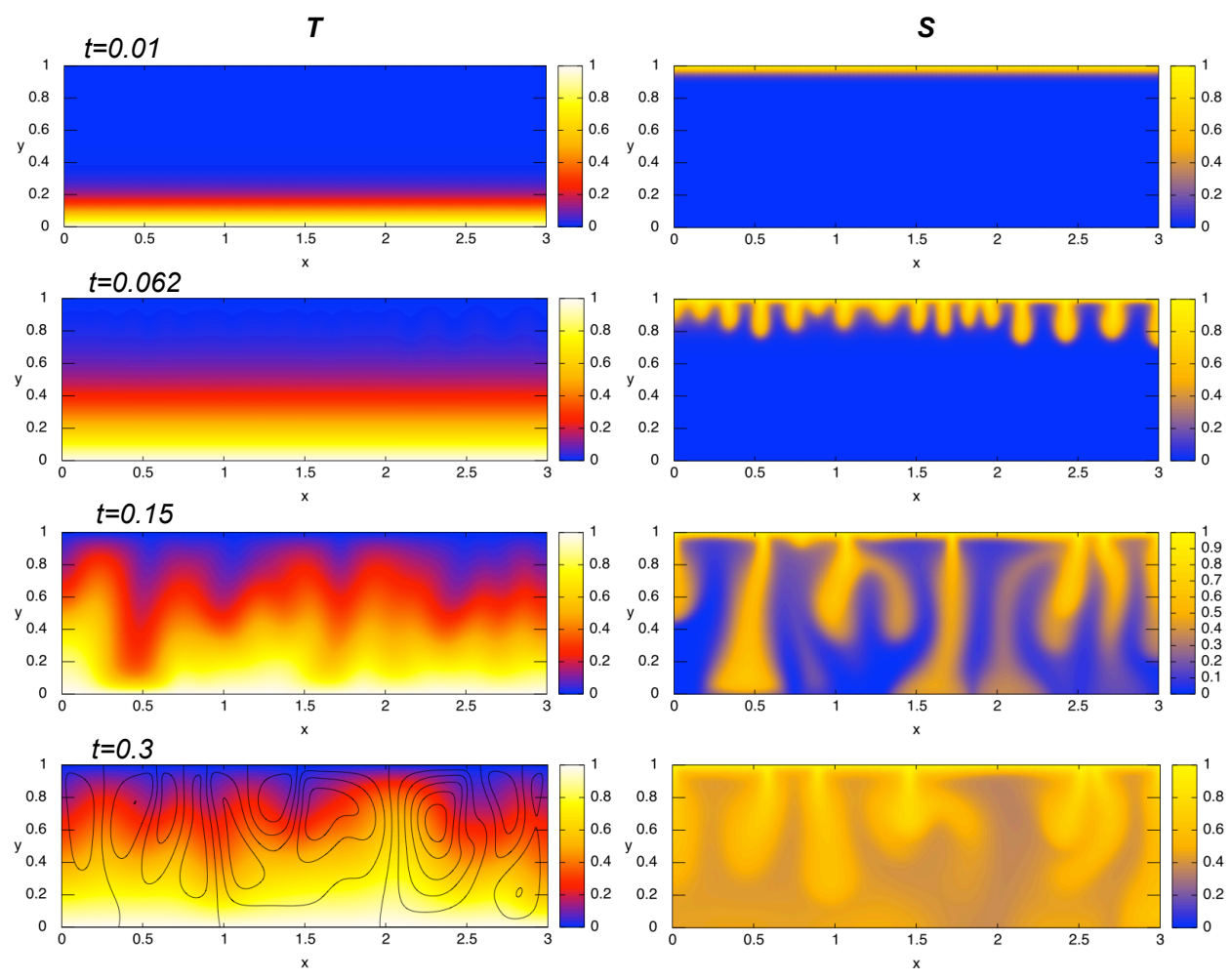

Figure 9: Concentration and temperature fields for $N=-10, R a=10$, and $\theta=0^{\circ}$. Streamlines are included in the most developed stage.

time $(t=0.3)$ the average concentration in the cavity reaches about $50 \%$ with a multicellular convective pattern controlled by the downwelling mass flow.

As the Rayleigh number and the inclination angle are increased the onset of convection occurs earlier. This onset is characterize by smaller scale fingering and by the development of a large downwelling at the left boundary $(x=0)$. This preferred flow path remains throughout the entire simulation time giving rise to zonation of mass concentration in two opposite corners of the porous enclosure. This convective pattern is accompanied by the formation of two large thermal plumes $(t=0.05)$ unlike the horizontal case.

With regard to high buoyancy forces $(N=-100)$, for the horizontal porous medium (Figure 11, video format available as supplementary material), the 

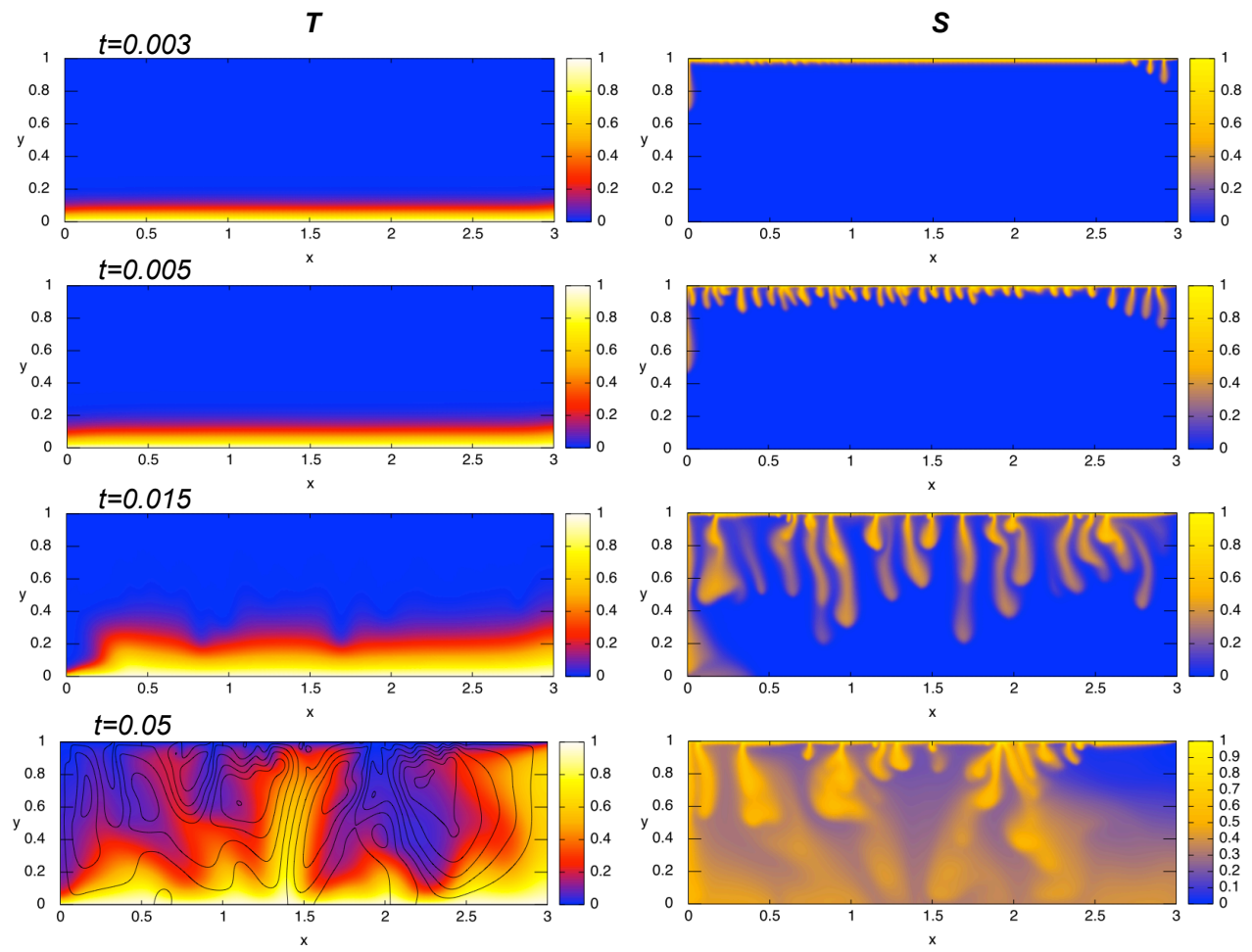

Figure 10: Concentration and temperature fields for $N=-10, R a=50$, and $\theta=15^{\circ}$.

onset of convection is characterized by small scale fingering with a short diffusive stage. Downwelling mass flow is characterized by multiple fingers that remain throughout the simulation time. In this case about $50 \%$ of average concentration has been attained at $t=0.02$. The flow pattern at this time consists of a complex arrangement of convective cells. On the basis that this flow pattern is highly dynamic, it explains the strong oscillatory character of $N u$ at these stages of the simulation.

Finally, in a combination of high $N$ with an inclination angle $\theta=15^{\circ}$ (Figure 12 , video format available as supplementary material), the onset of convection at the upper boundary is characterized by an uneven distribution, with the formation and development of downwelling mass flow for small and moderate values of $x$, whereas the remaining section of the boundary is still in a diffusive 

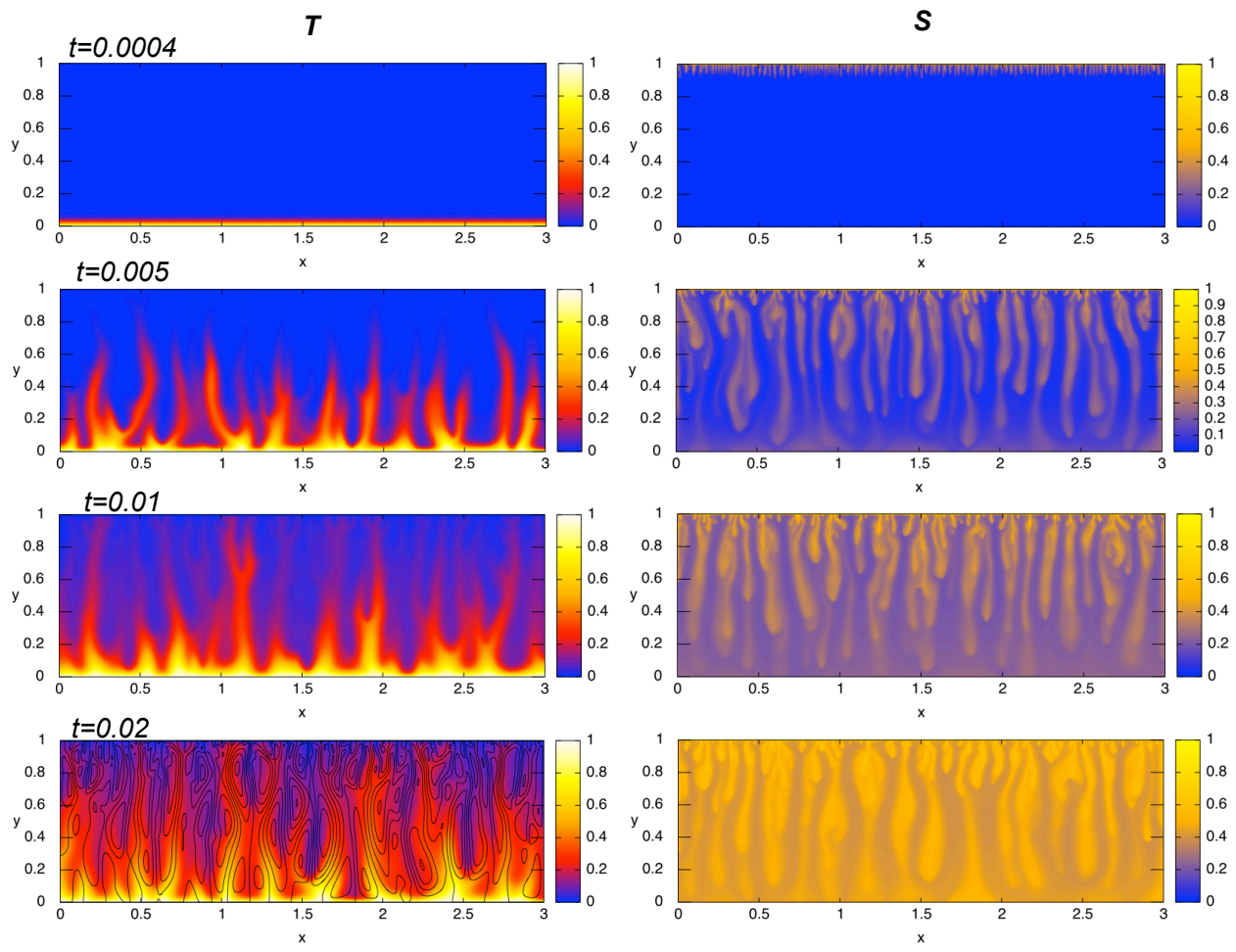

Figure 11: Concentration and temperature fields for $N=-100, R a=50$, and $\theta=0^{\circ}$. A video format of this figure is available online as supplementary material.

stage or in an early stage of convective transport. The preferential direction of flow controlled by $\theta$ leads to an accelerated saturation in a zone of small $x$ and $y$ with high temperature gradients (heating zone), whereas the opposite corner of the cavity becomes a low saturation zone accompanied by a strong thermal upwelling (cooling zone).

\section{Conclusion}

To gain further physical understanding of the problem of underground $\mathrm{CO}_{2}$ dissolution, we conducted transient numerical simulations to assess the convective mass and heat transport in a porous medium heated from below, salted from above, and subject to an inclination angle. We focused in a set of gov- 

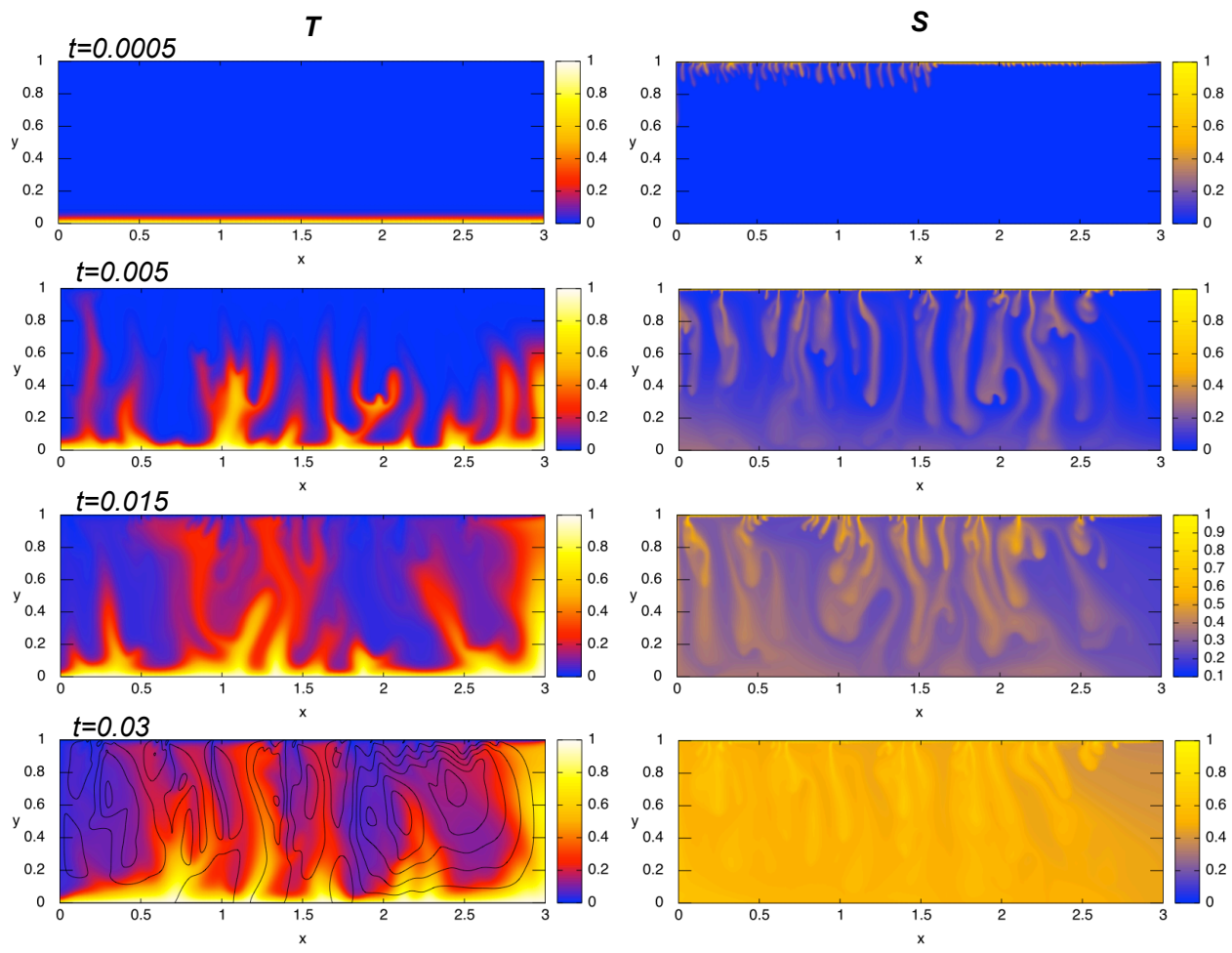

Figure 12: Concentration and temperature fields for $N=-100, R a=50$, and $\theta=15^{\circ}$. A video format of this figure is available online as supplementary material.

erning parameters given by two buoyancy ratios: $N=-10, N=-100$; three Rayleigh numbers: $R a=10, R a=50$, and $R a=80$; and four inclination angles: $\theta=0^{\circ}, 5^{\circ}, 10^{\circ}$, and $15^{\circ}$. Our results provided a quantitative insight on how mixing is enhanced as the buoyancy forces are increased. We also described the potential consequences that $\theta$ can have on the rate of solute mixing and in the form that onset of convection (fingering) takes place. Finally, the implications that $\theta$ can have on the flow patterns and heat transfer properties were identified. The concluding remarks are summarized in the following key points:

- Even though the Rayleigh number and the buoyancy ratio considerably speed up the saturation of the fluid with the solute, the inclination angle has a minor effect. Furthermore, a slight decrease in the mixing rate can 
occur when increasing $\theta$ as a consequence of preferential flow paths that force the solute flow through high saturation zones in the porous medium.

- For high buoyancy ratios, the inclination of the porous medium has the consequence of an uneven onset of convection at the boundary, being the most stable part that in the opposite direction to the gravitational force component.

- On the basis that thermal diffusivity is higher than mass diffusivity ( $L e=$ 10 in all the simulations), convective heat transport is controlled by convective mass transport in early stages of dissolution. Thermal upwellings adopt the shape of low solute concentration plumes. With regard to $\theta$, the heat transfer properties of the cavity $(N u)$ tend to increase with the inclination, since the convective patterns allow less but more intense thermal upwellings.

These concluding remarks help improve our understanding of fundamental behavior of double diffusive convection in porous media in the context of solute dissolution. We envisage further work that takes into account other important effects such as surface tension in the upper boundary (which is important for the case of miscible fluids) and heterogeneities.

\section{Acknowledgement}

We are thankful to anonymous reviewers for valuable comments to our final draft. We thank Dr. Luis Miguel de La Cruz for his support in computational modeling. The first author is thankful to DGAPA UNAM Research Program, and DGTIC UNAM for providing computational facilities. 
[1] S. Bouzgarrou, H. S. Harzallah, K. Slimi, Unsteady Double Diffusive Natural Convection in Porous Media-Application to $\mathrm{CO}_{2}$ Storage in Deep Saline Aquifer Reservoirs, in: Salame, C and Khoury, G and Aillerie, M (Ed.), Terragreen 13 International Conference 2013 - Advancements in Renewable Energy and Clean Environment, volume 36 of Energy Procedia, TerraGreen, 2013, pp. 756-765. doi:\{10.1016/j.egypro.2013.07.088\}, TerraGreen International Conference on Advancements in Renewable Energy and Clean Environment, Beirut, LEBANON, FEB 15-17, 2013.

[2] A. W. Islam, M. A. R. Sharif, E. Carlson, Numerical investigation of double diffusive natural convection of $\mathrm{CO}_{2}$ in a brine saturated geothermal reservoir, Geothermics 48 (2013) $101-111$.

[3] H. Emami-Meybodi, H. Hassanzadeh, C. P. Green, J. Ennis-King, Convective dissolution of $\mathrm{CO}_{2}$ in saline aquifers: Progress in modeling and experiments, International Journal of Greenhouse Gas Control 40 (2015) 238 - 266. Special Issue commemorating the 10th year anniversary of the publication of the Intergovernmental Panel on Climate Change Special Report on $\mathrm{CO}_{2}$ Capture and Storage.

[4] J. L. Bischoff, R. J. Rosenbauer, Salinity variations in submarine hydrothermal systems by layered double-diffusive convection, Journal of Geology 97 (1989) 613-623.

[5] M. J. Colçao, G. S. Dulikravich, Solidification of double-diffusive flows using thermo-magneto-hydrodynamics and optimization, Materials and Manufacturing Processes 22 (2007) 594-606.

[6] Y. Hao, J. J. Nitao, T. A. Buscheck, Y. Sun, Double-diffusive natural convection in a nuclear waste repository, Nuclear Technology 163 (2008) 38-46. International High-Level Radioactive Waste Management Conference, Las Vegas, NV, APR 30-MAY 04, 2006.

[7] D. A. Nield, Onset of thermohaline convection in a porous medium, Water Resources Research 4 (1968) $101-111$. 
[8] J. W. Taunton, T. Green, E. N. Lightfoot, Thermohaline instability and salt fingers in a porous medium, Physics of Fluids 15 (1972) $748-753$.

[9] O. V. Trevisan, A. Bejan, Mass and heat-transfer by high rayleigh number convection in a porous-medium heated from below, International Journal of Heat and Mass Transfer 30 (1987) 2341-2356.

[10] N. D. Rosenberg, F. Spera, Thermohaline convection in a porous-medium heated from below, International Journal of Heat and Mass Transfer 35 (1992) 1261-1273.

[11] D. K. Lin, Unsteady natural-convection heat and mass-transfer in a saturated porous enclosure, Warme und Stoffubertragung - Thermo and Fluid Dynamics 28 (1993) 49-56.

[12] M. Mamou, P. Vasseur, E. Bilgen, Multiple solutions for double-diffusive convection in a vertical porous enclosure, International Journal of Heat and Mass Transfer 38 (1995) 1787-1798.

[13] M. Sen, P. Vasseur, L. Robillard, Multiple steady-states for unicellular natural-convection in an inclined porous layer, International Journal of Heat and Mass Transfer 30 (1987) 2097-2113.

[14] F. J. Guerrero-Martínez, N. Karimi, E. Ramos, Numerical modeling of multiple steady-state convective modes in a tilted porous medium heated from below, International Communications in Heat and Mass Transfer 92 (2018) $64-72$.

[15] M. Mamou, P. Vasseur, E. Bilgen, Double-diffusive convection instability in a vertical porous enclosure, Journal of Fluid Mechanics 368 (1998) 263-289.

[16] I. Sezai, A. A. Mohamad, Three-dimensional double-diffusive convection in a porous cubic enclosure due to opposing gradients of temperature and concentration, Journal of Fluid Mechanics 400 (1999) 333-353. 
[17] P. Nithiarasu, T. Sundararajan, K. N. Seetharamu, Double-diffusive natural convection in a fluid saturated porous cavity with a freely convecting wall, International Communications in Heat and Mass Transfer 24 (1997) 1121-1130.

[18] F. Y. Zhao, D. Liu, G. F. Tang, Free convection from one thermal and solute source in a confined porous medium, Transport in Porous Media 70 (2007) 407-425.

[19] F. Y. Zhao, D. Liu, G. F. Tang, Natural convection in a porous enclosure with a partial heating and salting element, International Journal of Thermal Sciences 47 (2008) 569 - 583.

[20] N. Hadidi, R. Bennacer, Three-dimensional double diffusive natural convection across a cubical enclosure partially filled by vertical porous layer, International Journal of Thermal Sciences 101 (2016) 143-157.

[21] R. D. Jagadeesha, B. M. R. Prasanna, M. Sankar, Double diffusive convection in an inclined parallelogrammic porous enclosure, Procedia Engineering 127 (2015) 1346 - 1353. International Conference on Computational Heat and Mass Transfer (ICCHMT) - 2015.

[22] A. Riaz, M. Hesse, H. Tchelepi, F. Orr, Onset of convection in a gravitationally unstable diffusive boundary layer in porous media, Journal of Fluid Mechanics 548 (2006) 87-111.

[23] A. C. Slim, T. Ramakrishnan, Onset and cessation of time-dependent, dissolution-driven convection in porous media, Physics of Fluids 22 (2010) 124103.

[24] M. Szulczewski, R. Juanes, The evolution of miscible gravity currents in horizontal porous layers, Journal of Fluid Mechanics 719 (2013) 82-96.

[25] A. W. Islam, H. R. Lashgari, K. Sephernoori, Double diffusive natural convection of $\mathrm{CO}_{2}$ in a brine saturated geothermal reservoir: Study of non- 
modal growth of perturbations and heterogeneity effects, Geothermics 51 (2014) 325-336.

[26] A. Islam, A. K. N. Korrani, K. Sepehrnoori, T. Patzek, Effects of geochemical reaction on double diffusive natural convection of $\mathrm{CO}_{2}$ in brine saturated geothermal reservoir, International Journal of Heat and Mass Transfer 77 (2014) $519-528$.

[27] E. B. Soboleva, Density-driven convection in an inhomogeneous geothermal reservoir, International Journal of Heat and Mass Transfer 127 (2018) 784 -798 .

[28] S. Mrityunjay, A. Chaudhuri, P. H. Stauffer, R. J. Pawar, Simulation of gravitational instability and thermo-solutal convection during the dissolution of $\mathrm{CO}_{2}$ in deep storage reservoirs, Water Resources Research 56 (2020) e2019WR026126.

[29] D. A. Nield, A. Bejan, Convection in Porous Media, 4th ed., Springer, New York, 2013.

[30] F. J. Guerrero-Martínez, P. L. Younger, N. Karimi, Three-dimensional numerical modeling of free convection in sloping porous enclosures, International Journal of Heat and Mass Transfer 98 (2016) 257-267.

[31] F. J. Guerrero-Martínez, P. L. Younger, N. Karimi, S. Kyriakis, Threedimensional numerical simulations of free convection in a layered porous enclosure, International Journal of Heat and Mass Transfer 106 (2017) 1005-1013.

[32] H. K. Versteeg, W. Malalasekera, An Introduction to Computational Fluid Dynamics, The Finite Volume Method, Prentice Hall, 1995.

[33] E. Báez, A. Nicolás, 2D natural convection flows in tilted cavities: Porous media and homogeneous fluids, International Journal of Heat and Mass Transfer 49 (2006) 4773-4785. 